\title{
Obstruction of the Superior Mesenteric Artery Due to Emboli from the Thoracic Aorta in a Patient with Thromboangiitis Obliterans
}

\author{
Chiaki Kamiya, MD, ${ }^{1}$ Juno Deguchi, MD, PhD, ${ }^{1}$ Tadashi Kitaoka, MD, ${ }^{1}$ \\ Jun Suzuki, MD, PhD, ${ }^{1}$ Keiko Abe, MD, PhD, ${ }^{2}$ and Osamu Sato, MD, PhD ${ }^{1}$
}

\begin{abstract}
A 48-year-old woman presented at our hospital with acute abdominal pain 3 years after being diagnosed with thromboangiitis obliterans (TAO). Computed tomography revealed occlusion of the superior mesenteric artery (SMA) and multiple kidney infarction with thrombus floating in the thoracic aorta connected with the intercostal artery. Despite emergency embolectomy, further thromboembolism eventually required massive resection of the intestine with jejunostomy and colostomy and permanent intravenous hyper-alimentation therapy. Although TAO rarely involves the large artery, the aorta could be the source of embolization in patients with TAO.
\end{abstract}

Keywords: thromoboangiitis obliterans, superior mesenteric artery obstruction

\section{Introduction}

Thromboangiitis obliterans (TAO), also known as Buerger's disease, is a non-atherosclerotic inflammatory disease characterized by thrombotic occlusion of the small or medium arteries or superficial veins in the upper and lower extremities. ${ }^{1)}$ This disease classically presents in young male smokers, although the prevalence of female patients has increased over the last three decades. $\left.{ }^{2}\right)$ The etiology remains elusive; although several factors including hygiene and genetic predisposition contribute to the development of this disease. In addition to the involvement of tobacco use in the pathogenesis and progression, patients with TAO usually have enhanced platelet function or endothelial dysfunction, which precipitates thrombotic formation in affected vessels. The disease usually

${ }^{1}$ Department of Vascular Surgery, Saitama Medical Center, Saitama Medical School, Kawagoe, Saitama, Japan

${ }^{2}$ Department of Pathology, Saitama Medical Center, Saitama

Medical School, Kawagoe, Saitama, Japan

Received: March 7, 2014; Accepted: May 12, 2014

Corresponding author: Juno Deguchi, MD, PhD. Department of

Vascular Surgery, Saitama Medical Center, Saitama Medical

School, 1981 Kamoda, Kawagoe, Saitama 385-5330, Japan

Tel: +81-49-228-3400, Fax: +81-49-228-3462

E-mail: jdegu-tky@umin.ac.jp presents as ischemia of the distal extremities, and might initially affect arteries in the peripheral portion of a single limb before progressing to proximal or multiple arteries.

Thromboangiitis obliterans rarely involves larger arteries such as the iliac or visceral arteries. Several reports have described that acute mesenteric artery occlusion develops in some patients with TAO and leads to life-threating or fatal events. ${ }^{3-6)}$ These reports noted that the mesenteric arteries with vasculitis are responsible for local thrombus formation, although embolism of the mesenteric arteries could not always be ruled out because of the absence of pathological data. Acute intestinal ischemia can result from the embolic occlusion of the mesenteric artery. Alpaslan, et al. described a patient with TAO who had large thrombus in the main pulmonary artery that precipitated right heart failure, suggesting that a large artery could be an embolic source in TAO.

We described the thromboembolism from the thoracic aorta that obstructed the superior mesenteric artery (SMA), and subsequently caused acute intestinal ischemia in a female patient with TAO. To the best of our knowledge, this is the first report of an embolism causing intestinal ischemia in a patient with TAO. 

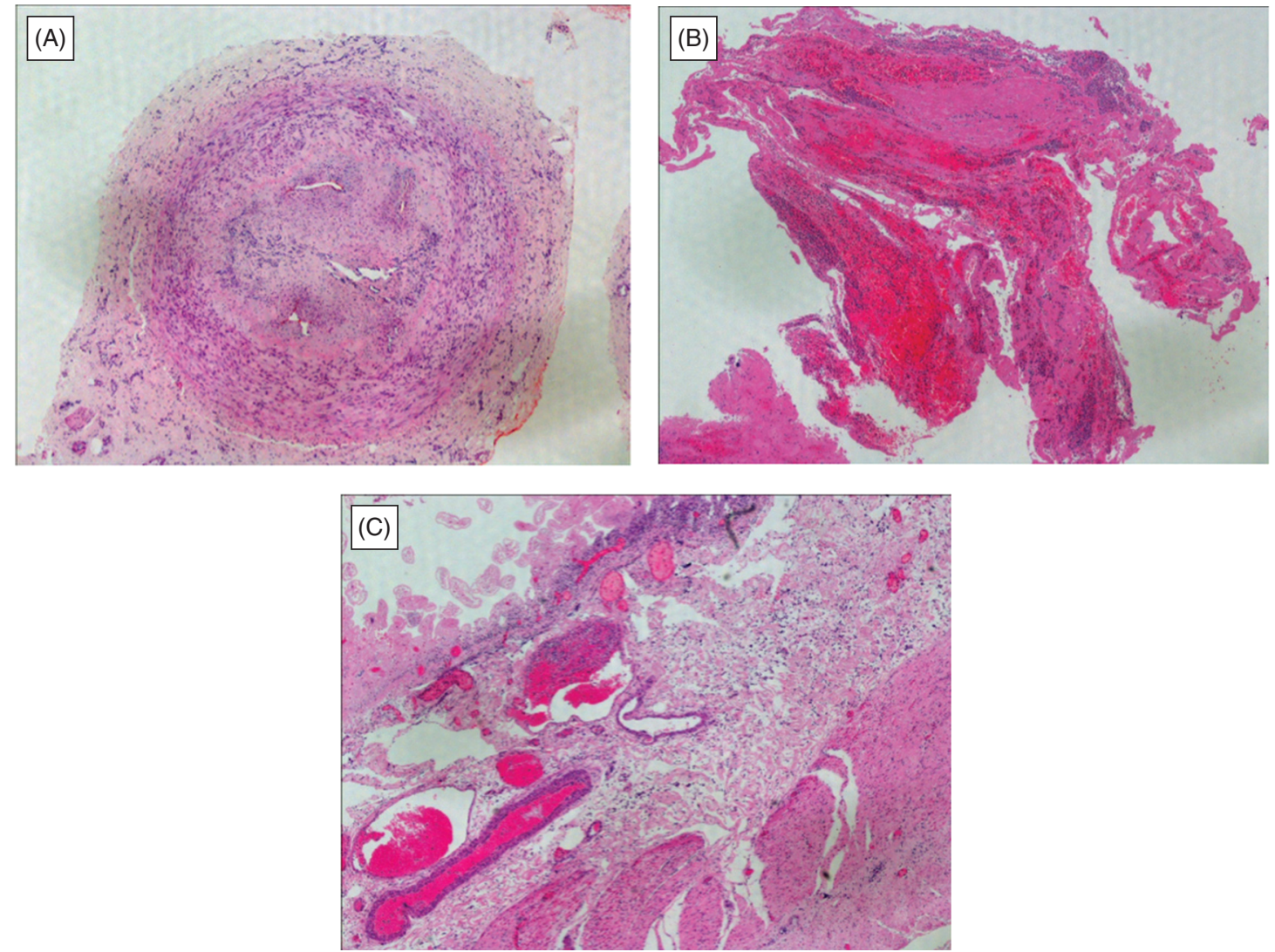

Fig. 1 Histological findings of surgical specimens from the patient. (A) Posterior tibial artery from amputated leg contained organized thrombus. Although proliferation of fibroblasts and capillary structures with lymphocytic infiltration were totally obstructed inside of the artery, either inner or outer elastic laminae of the medial layer was intact, which distinguish thromboangiitis obliterans (TAO) for arteriosclerosis and other systemic vasculitides. Hematoxylin and eosin staining. (B) The resected specimen by embolectomy of the SMA. It comprised organized emboli mixed with fresh thrombi. Note that neutrophils infiltrates into clots. Hematoxylin and eosin staining. (C) Mucosal layer of the small intestine was extensively necrotic and villous structures were left out. Prominent congestion and edema were observed in the submucosa. There were not observed any finding of vasculitis. Hematoxylin and eosin staining, $\times 40$.

\section{Case Report}

A 48-year-old Japanese woman who had endured severe upper abdominal pain that had persisted over $6 \mathrm{~h}$ arrived at our hospital by ambulance.

She experienced severe ischemia of the right foot three years before presentation of abdominal pain. She had no risk factors for atherosclerosis, despite smoking one pack of cigarette daily. Laboratory findings were negative for autoimmune antibodies including anti-phospholipid antibody. She had hyperhomocysteinemia, but no history of disordered coagulation diseases such as protein $\mathrm{C}$ or protein $\mathrm{S}$ deficiency. Antithrombin III levels were within normal range. She underwent femoral-tibial arterial bypass surgery to rescue the right lower extremity; however, fresh recurrent thrombi resulted in graft occlusion and eventual amputation of the right lower extremity below the knee. Microscopic examination of specimens from the amputated leg revealed thromoboangiitis obliterans (Fig. 1A). Thereafter, she quit smoking completely and was treated with a $5-\mathrm{HT}_{2}$ blocker for vasodilation without specific antiplatelets.

She remained stable for three years. When she arrived at our hospital with severe upper abdominal pain developed for over $6 \mathrm{~h}$.

The findings upon admission included blood pressure of $170 / 92 \mathrm{mmHg}$ and sinus rhythm determined by electrocardiography of 94 beats/min. Abdominal computed tomography (CT) in a hospital which referred to us, had identified the occluded SMA and 
(A)
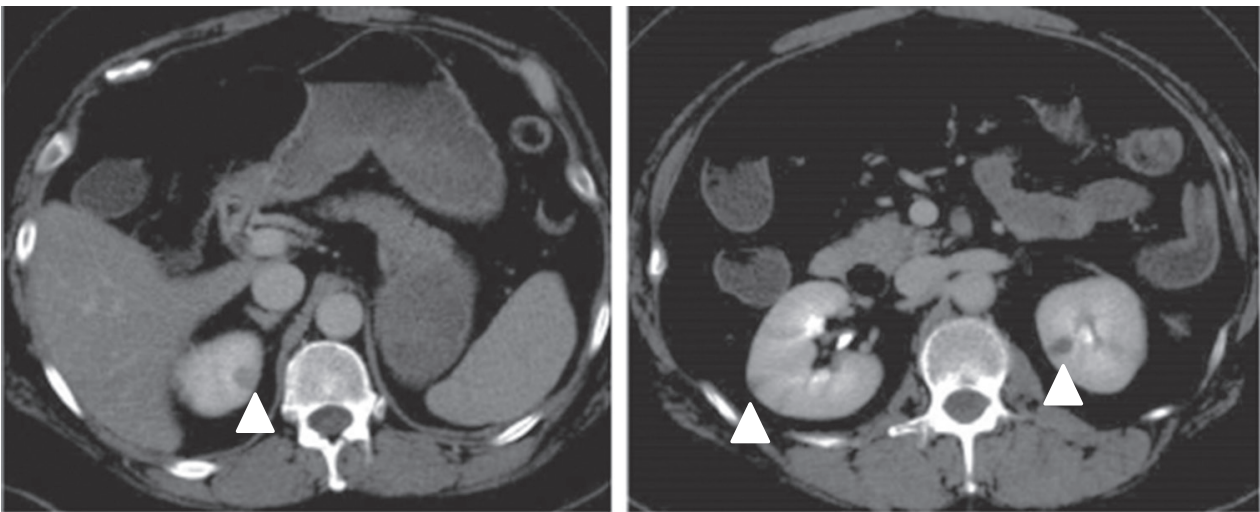

(B)

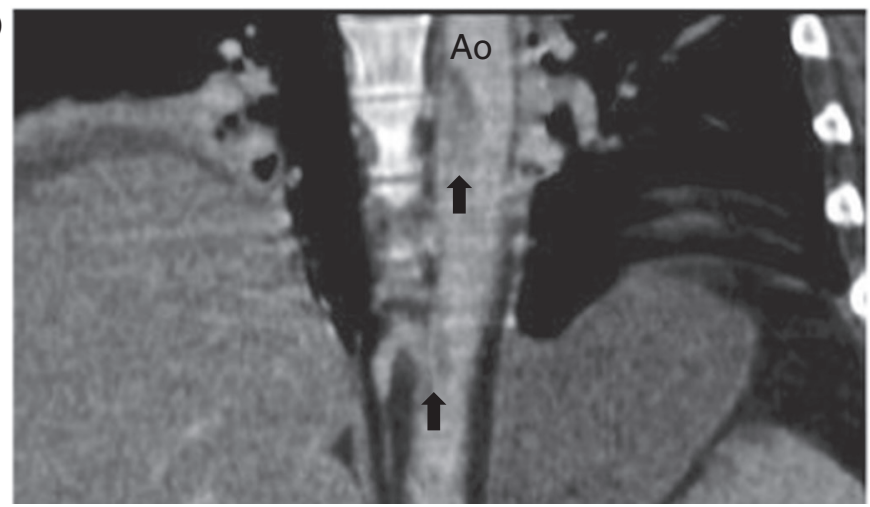

Fig. 2 Computed tomography (CT) revealed thrombi in the descending thoracic aorta and multiple infarctions in the spleen and kidney. (A) Cross-sectional CT images showed multiple kidney infarctions (white arrowheads). (B) A sagittal CT image showed floating thrombus in the descending thoracic aorta (black arrows). Ao: aorta.

multiple small kidney infarctions, as well as the floating thrombi in the thoracic aorta with neither aortic aneurysmal nor atheroma lesions (Fig. 2). The left atrium and ventricle were free of thrombi. Laboratory findings showed a white blood cell count of $3.42 \times 10^{4} / \mu \mathrm{L}$, with elevated d-dimer $(5.57 \mu \mathrm{g} / \mathrm{mL})$. Acute obstruction of the SMA was diagnosed, and thus the patient underwent emergency laparotomy. The intestine had not yet become grossly necrotic; therefore, embolectomy of the SMA proceeded using a Fogarty catheter. The resected specimen comprised organized emboli mixed with fresh thrombi (Fig. 1B). We completed the surgical procedure on a pulsatile SMA without resection of the intestine. However, acidosis developed $24 \mathrm{~h}$ after the first laparotomy. A repeat CT revealed a re-occluded SMA with intestinal ischemia and a new spleen infarction. A second emergency laparotomy revealed necrosis almost entire small intestine and the right side of the colon. We resected the necrotic intestine and colon and prepared a jejunostomy and a transverse colostomy. Resected specimens showed extensive mucosal necrosis and congestion of the intestinal wall, suggesting of occlusive thrombus in the SMA. Additionally, vasculitis was not observed (Fig. 1C). Analysis of first CT images afterward showed large floating thrombi in the thoracic aorta connected to the intercostal artery (Fig. 3). The patient received anticoagulation therapy. We once considered thrombectomy in the aorta, but she was followed closely because of effective anticoagulation therapy. The postoperative course was uneventful except for transient liver dysfunction. However, the massive intestinal resection required her to receive permanent intravenous hyper-alimentation therapy, so she was transferred to another hospital for rehabilitation, 28 days after surgery. Follow-up CT, six month later confirmed the absence of thrombus in the thoracic aorta. 

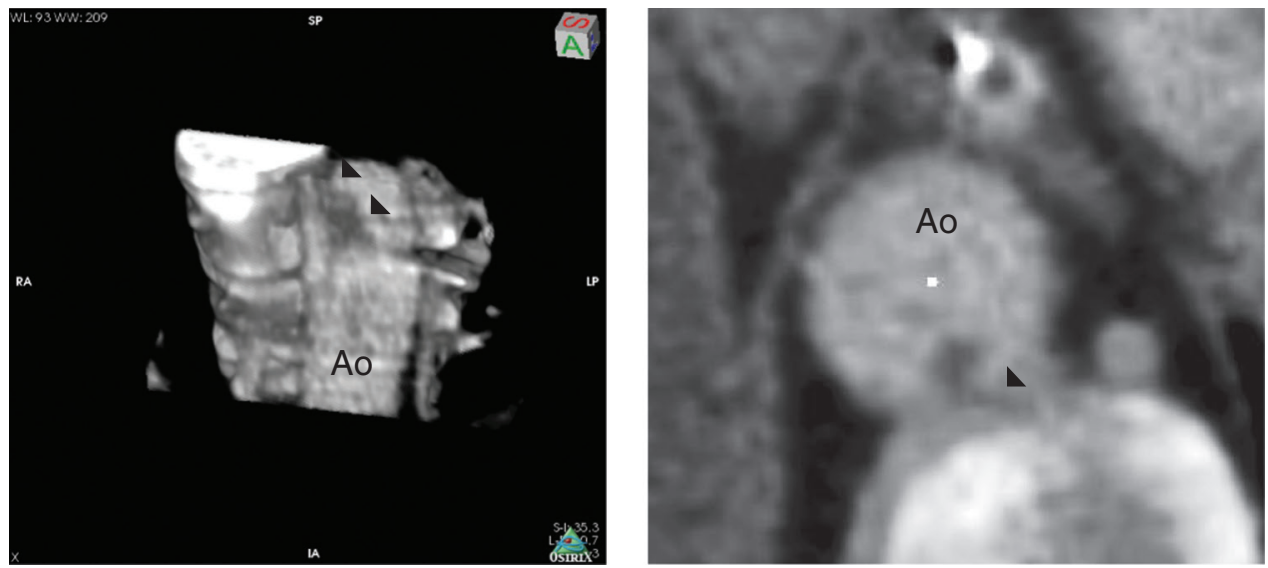

Fig. 3 Computed tomography (CT) images of floating thrombus in the thoracic aorta. Left panel shows a three dimensional CT image and right shows a cross-sectional view of the thrombus. Note that thrombus in the thoracic aorta connecting to the intercostal artery (black arrowheads). Ao: aorta.

\section{Discussion}

TAO involves the infrapopliteal arteries in general; however, it is infrequently associated with larger arteries including the mesenteric, colonic and coronary arteries. There has been reported intestinal necrosis due to obstruction of the mesenteric artery in patients with TAO. Histopathological findings would be necessary to diagnose as thrombotic complication of TAO in such unusual locations; however, most reports do not mention histopathology specimen of the mesenteric artery. Considering that embolization causes symptoms that mimic of those of TAO, embolization might induce acute mesenteric arterial occlusion even in some patients with TAO like common patients with atrial fibrillation. We excluded atrial fibrillation, atherosclerosis and autoimmune disease from the present patient. However, thrombi in the thoracic aorta and the acute mesenteric occlusion developed in our patient. Thrombi in the thoracic aorta probably caused acute mesenteric occlusion. This notion is supported by the fact that surgical specimens of the superior mesenteric artery (SMA) from the first laparotomy of our patient comprised emboli mixed with fresh thrombi. Furthermore, SMA occlusion as well as kidney and spleen infarction was evident, as were sinus heart rhythms with no aneurysm or atherosclerosis in the thoracic aorta. However, the cause of thrombi in the thoracic aorta was elusive. We speculate that thrombi in the thoracic aorta were associated with TAO. Indeed thoracic aorta involvement in TAO has not been reported. However, Alpaslan, et al.7) described a patient with TAO who had large thrombus in the main pulmonary artery that precipitated right heart failure.

Hyperhomocysteinemia is an independent risk factor for vascular occlusive disease. It is considered to arise via the same mechanism of atheroma that develops from accumulating conjoined homocysteine and lipoprotein, and disrupts endothelial cell function. Kenari, et al. recently, reported that patients with TAO had higher plasma concentration of homocysteine and was associated with thrombosis. ${ }^{8)}$ Moreover, Courtney, et al. described a young woman with hyperhomocysteinemia and TAO who lost an ischemic leg and subsequently developed fatally small bowel infarction by local thrombosis. ${ }^{9)}$ The present patient did not have hypercholesterolemia and we did not measure the serum lipoprotein level. The precise relationship between TAO and hyperhomocysteinemia remains unclear.

We speculated that thrombi-formed in the intercostal artery and extended into the thoracic aorta where they became shredded and caused the SMA occlusion in our patient. Only one report published in Japanese has described a young patient with TAO who underwent abdominal aortic bypass surgery and had solitary thrombus in the 5th lumber artery. ${ }^{10)}$ 


\section{Conclusion}

We described a patient with TAO and SMA occlusion by the emboli from the thoracic aorta. Thrombi should be explored in large artery such as the aortas in patients with TAO who present with acute abdominal pain.

\section{Disclosure Statement}

Each author of this article has no conflict of interest such as employment, leadership position, advisory role, stock ownership, patent royalties or honoraria. Each author has no sponsor that provided financial support for this study.

\section{References}

1) Olin JW. Thromboangiitis obliterans (Buerger's disease). N Engl J Med 2000; 343: 864-9.

2) Sasaki S, Sakuma M, Kunihara T, et al. Current trends in thromboangiitis obliterans (Buerger's disease) in women. Am J Surg 1999; 177: 316-20.

3) Kobayashi M, Kurose K, Kobata T, et al. Ischemic intestinal involvement in a patient with Buerger disease: case report and literature review. J Vasc Surg 2003; 38: 170-4.

4) Cho YP, Kwon YM, Kwon TW, et al. Mesenteric Buerger's disease. Ann Vasc Surg 2003; 17: 221-3.

5) Cho YP, Kang GH, Han MS, et al. Mesenteric involvement of acute-stage Buerger's disease as the initial clinical manifestation: report of a case. Surg Today 2005; 35 : 499-501.

6) Iwai T. Buerger's disease with intestinal involvement. Int J Cardiol 1998; 66 Suppl 1: S257-63; discussion S265.

7) Alpaslan M, Akgün G, Düven O, et al. Thrombus in the main pulmonary artery of a patient with thromboangiitis obliterans: observation by transthoracic echocardiography. Eur J Echocardiogr 2001; 2: 139-40.

8) Kenari AY, Karimi M, Salimi J, et al. Plasma levels of homocysteine in Buerger's sufferers and healthy heavy smokers and non-smokers. Clin Lab 2013; 59: 93-6.

9) Courtney PA, Sharpe PC, Lee RJ. Homocysteine and “Buerger's disease". Postgrad Med J 2002; 78: 500-5.

10) Oshima A, Ueno A. A young male with buerger's disease who had the solitary clot formation in the aorta. In: Mishima Y ed. Special diseases designated by Ministry of Health and Welfare of Japan. Research report. Survey research group of systematic vascular disease. 1985: 635 . 